12 (30.7\%) had hypoactive labyrinth (bilateral or unilateral) and one $(0.03 \%)$ had hyperactive labyrinth (bilateral or unilateral). Two children (0.05\%) had abnormal subjective visual vertical. Among the various diagnoses made, the four main pathology found in our study were vestibular migraine in 17 (43.5\%), vasovagal syncope in six (15.4\%), otolithic dysfunction in three $(7.7 \%)$, and posttraumatic concussion in three $(7.7 \%)$ patients.

Conclusion In our study, we have found that the prevalence of pediatric giddiness to be $0.007 \%$ and the most common diagnosis made was vestibular migraine. It is quite feasible and essential to evaluate children with vertigo and dizziness systematically to make a relevant clinical diagnosis, which helps in the proper management of these patients and also allay anxiety in parents.

\section{A0011: Microsurgical Anatomy of Stapedius Muscle: Anatomy Revisited, Redefined with Potential Impact in Surgeries \\ Harshita T.R., ${ }^{1}$ K. C. Prasad ${ }^{2}$ \\ ${ }^{1}$ Sri Devaraj Urs Medical College, Kolar, Karnataka, India ${ }^{2}$ Department of ENT, Sri Devaraj Urs Medical College, Kolar, Karnataka, India}

Stapedius muscle, even though being the smallest skeletal muscle in human body, has a major role in otology. As many of the distinguished books in otology missed to explain much about stapedius muscle, and also considering the need for the anatomy-based visit to this small muscle, we felt it was important to have an exercise like this. In the dissection hall of our institution, we dissected 32 cadaveric temporal bones and delineated stapedius muscle as a part of PG teaching program to have a clear idea of the anatomy of the stapedius muscle, its origin, attachment, extension, and size (all dimensions). Length of the stapedius muscle varied between 9 and $11 \mathrm{~mm}$, stapedius tendon measured approximately $2 \mathrm{~mm}$. The muscle had a classical sickle shape with tendon looking like a handle of the sickle. It has a bulky belly with a maximum breadth of 1 to $3 \mathrm{~mm}$. Why to have a clear idea about the anatomy of the stapedius muscle is that, unless the anatomy is clear, there is chance of confusing the muscle with facial nerve while doing facial nerve grafting and also while drilling for facial nerve decompression, even in experienced hands who may get confused and decompress the muscle. Stapedius muscle is said to be the smallest in the body but not as small as its been described. Detailed awareness of the anatomy of stapedius muscle is needed so as to avoid confusion while facial nerve grafting and while drilling.

\section{A0012: Simultaneous Labyrinthectomy and Cochlear Implantation \\ Joyce Rozario ${ }^{1}$ \\ ${ }^{1}$ Narayana Health City, Bengaluru, Karnataka, India}

Introduction Reports indicate that the cochlea remains responsive to electrical stimulation following labyrinthectomy. Post-stapedotomy surgery complications like persistent vertigo and sensorineural hearing loss are debilitating. This case describes the management of a patient by simultaneous labyrinthectomy and cochlear implantation following complications of stapedotomy.

Case Presentation A 37-year-old male teacher who had undergone stapedotomy 1 month ago at a local hospital, presented with complaints of right ear hearing loss and vertigo. Examination revealed a right-sided posterior marginal perforation and dislocated stapes piston with clear fluid seen filling the middle ear. Pure tone audiogram showed a right ear moderate to severe mixed hearing loss and a left moderate mixed hearing loss. A high-resolution computerized tomogram (HRCT) of the temporal bone was normal. Right revision stapedotomy, closure of perilymph leak and myringoplasty were done. Patient improved symptomatically with improvement of hearing and relief from vertigo. However, 3 months later patient developed viral labyrinthitis with severe vertigo and right-sided severe profound hearing loss. He was treated conservatively with IV antibiotics. Patient continued to have intractable vertigo and hearing loss. The patient subsequently underwent right simultaneous labyrinthectomy and cochlear implantation 7 months after the second surgery. Postoperatively, the patient had dramatic relief from vertigo and was able to resume to his daily routine activities.

Discussion and Conclusion Simultaneous labyrinthectomy and cochlear implantation following complications of stapedotomy offers successful treatment and is yet another indication for cochlear implantation.

\section{A0013: Outcomes of Endoscopic Stapedotomy Khageswar Rout}

Traditional surgery for otosclerosis is performed by microscopic approach. However, in recent years, endoscopic instrumentation, techniques and knowledge have greatly improved and, in our opinion, endoscopic stapedotomy will gain increasing importance in otology in future. It is a newer prospective. The aim of this presentation is to highlight importance of endoscope over microscope, particularly the panoramic view of entire foot area, both anterior crus and posterior crus visualization, and the results associated with endoscopic technique. We conducted a study on endoscopic stapedotomy between June 2016 and May 2018 in which we operated 16 cases. Preoperative surgical findings, complications, and duration of surgery, and air bone gap improvements were analyzed. Postoperative results were very good at par with microscopic technique.

\section{A0014: A New Method of Cadaver Dissection in the Temporal Bone}

M. Kumaresan, Navin Bharath

Aim Illustration of an easy method of entering into the middle ear and proceed further to the brain in a cadaver.

Methods Learning the live anatomy is essential for any surgery before it is undertaken by a surgeon. It can be undertaken in human cadaver or sheep cadaver. We dissected out the temporal mandibular joint as a first-step study. The external auditory canal, the whole length and breadth can be studied easily after removal of the thin bone between external auditory canal and temporal mandibular joint. 